\title{
Programas de formación en comunicación clínica: una revisión de su eficacia en el contexto de la enseñanza médica.
}

\section{"Clinical Communication teaching programs: a review in the context of medical education."}

Roger Ruiz Moral

Facultad de Medicina de Córdoba. Unidad Docente de Medicina de Familia de Córdoba.

Grupo Comunicación y Salud

Introducción: La comunicación clínica es una competencia del médico que influye en el proceso diagnóstico y terapéutico poderosamente y que puede y debe ser enseñada. Objetivo: Valorar la efectividad de programas y metodologías para la enseñanza de entrevista clínica en los ámbitos pre y postgraduado y en la educación médica continuada. Material y Métodos: Revisión narrativa de estudios experimentales sobre la formación en comunicación clínica, mediante búsquedas bibliográficas en las bases de datos Medline y Cochrane abarcando los diez últimos años o sin límite temporal y utilizando los siguientes descriptores: interviewing skills \& randomized controlled trial \& training; doctor-patient communication \& randomized.

Resultados: Un total de $\mathbf{2 4}$ artículos fueron aceptados y analizados, 11 en formación especializada, 8 en FMC y 5 en pregrado. La calidad metodológica fue mayor en los estudios más recientes y los de FMC. En los estudios con médicos los resultados positivos se suelen relacionar con buenos diseños, mediciones de conducta, cursos o seminarios intensivos en el contexto de rotaciones o prácticas clínicas relacionadas con el tema, uso de metodologías docentes interactivas que usan el feedback $y$, en estudiantes y residentes, con inicios tempranos en la formación.

Conclusiones: Para diseñar actividades formativas en comunicación clínica es preciso tener en cuenta los aspectos docentes que han mostrado su eficacia. La situación incipiente en nuestro pais de este tipo de formación hace prioritario aspectos como una capacitación del profesorado clínico para desarrollar programas básicos bien estructurados y adaptados a las condiciones locales en el marco de los principios de una enseñanza de adultos.

Palabras clave: comunicación médico-paciente,

formación médica continuada

Correspondencia:

Roger Ruiz Moral

Unidad Docente de Medicina de Familia

C/ Dr Blanco Soler, 2, 14004 Córdoba

Tel.: 957012544

E-mail: roger.ruiz.sspa@juntadeandalucia.es
Background: Clinical communication is a physician competence related with doctor diagnostic and therapeutic effectiveness. Clinical Communication can and ought to be teached.

Aim: To assess the effectiveness of clinical interviewing teaching programs in pre-postgraduate and CME settings. Material \& Methods: Narrative review of experimental studies in teaching communication skills. A bibliographic searching in Medline and Cochrane data bases with or without time boundaries and using the following descriptors: interviewing skills \& randomized controlled trial \& training; doctor-patient communication \& randomize was carried out.

Results: 24 papers were accepted and analysed, 11 in vocational training period, 8 in CME, and 5 with medical students. Metodological quality was higher in those studies more recents and in those from CME. Best designs, behavior as main outcome measured, intensive courses or seminars in the context of a clinical rotation related with the topic, adult and learned centred approaches with structured feedback and an early introduction in students and residents, were associated with positive results.

Conclusions: An evidence based approach is useful for planning future teaching strategies and methodologies in patient-physician relationship courses or seminars. The early stages in the development of this field in Spain recommend first a teachers teaching program, developing basic skills oriented and well structured programs, and to keep in mind local resources and a adult learning focused methodologies.

Key words: patient-physician communication, review, clinical interviewing, continuing medical education, training program.

\section{INTRODUCCIÓN}

La importancia de la comunicación médicopaciente ha sido reconocida desde hace tiempo como el elemento nuclear del acto médico ${ }^{1,2}$. Más recientemente, numerosos estudios de investigación han 
puesto de manifiesto como una comunicación clínica afecta positivamente al proceso diagnóstico-terapéutico $^{3}$. Por ello se ha reconocido la necesidad de enseñar y valorar las habilidades comunicacionales en las escuelas de medicina y durante el proceso de especialización y formación médica continuada. El Consenso de Toronto ${ }^{4}$ publicó en 1991 una revisión sobre la efectividad de la comunicación, las deficiencias más importantes que ocurrían en la práctica clínica y los métodos de enseñanza recomendados, introduciendo entonces el mensaje de que la comunicación clínica podía ser enseñada y evaluada. En 1993 el General Medical Council ${ }^{5}$ recomendó que las habilidades comunicacionales fuesen introducidas durante el pregrado en el Reino Unido y similares declaraciones han realizado la Association of American Medical Colleges por lo que respecta a este tipo de educación en Estados Unidos ${ }^{6}$ y en Canadá ${ }^{7}$. Posteriores Conferencias de Consenso ${ }^{8,9}$ se han ido desarrollando en las diferentes reuniones organizadas en los últimos años que finalmente han llevado a la creación de la European Association for Communication in Health Care (EACH) (Oxford, Julio 1996; Amsterdam, Junio 1998, Barcelona, Septiembre 2000 y Warwick (RU) Septiembre 2002) y que, entre otras misiones, han tratado de definir y ofrecer directrices sobre los programas de enseñanza y evaluación de la comunicación clínica. En España desde finales de los 80 un grupo de profesionales de la medicina y enfermería (El Grupo Comunicación y Salud) viene desarrollando este ámbito en todas sus dimensiones y facetas. Por otro lado, el Programa de la Especialidad de Medicina de Familia y Comunitaria ${ }^{10}$ contempla la entrevista clínica. como una tarea educativa que hay que desarrollar durante la formación de estos especialistas. Sin embargo, no ha sido hasta el momento presente cuando la Comisión Nacional de esta especialidad ha encargado a un grupo de trabajo relacionado con el Grupo Comunicación y Salud y el EACH ${ }^{11}$ el establecimiento de recomendaciones concretas sobre objetivos, contenidos y metodologías docentes. Por otro lado, la Consejería de Salud de la Junta de Andalucía trabaja actualmente en un programa de formación común para los especialistas en formación del sistema sanitario público de Andalucía ${ }^{12}$, que incluye un módulo específico de formación en comunicación médicopaciente, lo que supone un reconocimiento pionero de la importancia de este ámbito por parte de los proveedores de servicios institucionales en nuestro pais. Se ha progresado mucho desde el Consenso de Toronto, especialmente en lo que respecta a la inves- tigación en la efectividad de la comunicación clínica, su enseñanza y valoración y la creación de redes internacionales de asociaciones que está generando un cuerpo de conocimientos y experiencias compartidas de gran utilidad para el médico práctico. Sin embargo, actualmente existen aún muchos aspectos que precisan ser investigados con mayor profundidad y con nuevos enfoques entre ellos muchas de las recomendaciones que han sido propuestas.

El presente artículo pretende realizar una valoración crítica de los estudios experimentales que se han llevado a cabo para evaluar la efectividad de programas y metodologías para la enseñanza de entrevista clínica en el ámbito de la formación médica tanto de pregrado como en la formación especializada y en la educación médica continuada y en base a esto proponer algunas estrategias de implantación de la formación en comunicación en nuestro pais.

\section{MATERIAL Y MÉTODOS}

Este trabajo no es un metaanálisis sino una revisión narrativa de estudios experimentales sobre la formación en comunicación clínica. Para ello se han realizado varias búsquedas bibliográficas en las bases de datos Medline y Cochrane que abarcaron los diez últimos años o sin límite temporal. Se excluyeron aquellos artículos cuyo ámbito era la psiquiatría y la psicología abarcando cualquier otro ámbito clínico. Se utilizaron los siguientes descriptores : interviewing skills (búsqueda en los 10 últimos años), interviewing skills \& randomized controlled trial \& training (sin límite de tiempo); doctorpatient communication \& randomized ( $\sin$ límite de tiempo). Otra fuente importante de recuperación han sido las referencias de artículos disponibles. Un total de 24 artículos fueron aceptados y analizados. Las variables relacionadas con aspectos docentes que se compararon fueron las siguientes:

El número de participantes en la actividad formativa.

El tipo de curso: incluyendo el número de horas docentes, su distribución temporal (intensivo a tiempo completo o longitudinal compatibilizándolo con otras actividades - tiempo parcial-).

La actividad clínica que realizaban los estudiantes/médicos en el momento del curso.

La orientación docente predominante del curso (centrada en la experiencia del alumno o clásica -centrada en el profesor-).

El tipo y la cualificación de los docentes.

El año de residencia (en el caso de residentes).

Para valorar la calidad metodológica de los estu- 
Tabla 1. Evaluación de la calidad de los estudios

\begin{tabular}{|c|c|c|}
\hline \multicolumn{3}{|l|}{ CARACTERÍSTICAS } \\
\hline Estudio & $A$ & G C \\
\hline \multicolumn{3}{|l|}{ EN RESIDENCIA } \\
\hline Schubert (14) & 0 & 1 \\
\hline Smith (20) & 0 & 1 \\
\hline Breulin (23) & 0 & 2 \\
\hline THOMPSON (13) & 0 & 1 \\
\hline Merkel (22) & 0 & 2 \\
\hline Roter (18) & 2 & 2 \\
\hline Kauss (19) & 2 & 2 \\
\hline Smith (21) & 2 & 2 \\
\hline Gask (16) & 0 & 1 \\
\hline Smith (17) & 2 & 2 \\
\hline Ruiz Moral (15) & 2 & 2 \\
\hline \multicolumn{3}{|l|}{ En FMC } \\
\hline Bowman (31) & 0 & 1 \\
\hline Levinson (26) & 1 & 1 \\
\hline Inui (24) & 2 & 2 \\
\hline Evans (25) & 2 & 2 \\
\hline Girón (28) & 2 & 2 \\
\hline Roter (27) & 2 & 2 \\
\hline Muñoz (29) Ruiz (30) & 2 & 2 \\
\hline Sanci (32) & 2 & 2 \\
\hline
\end{tabular}

$\begin{array}{lll}\text { AP } & \text { S } & \text { AlT } \\ & & \\ 0 & 2 \\ 2 & 0 \\ 1 & 0 \\ 0 & 2 \\ 2 & 0 \\ 0 & 0 \\ 2 & 0 \\ 2 & 2 \\ 2 & 2 \\ 0 & 0 \\ 2 & 2 \\ & \\ 2 & 2 \\ 2 & 0 \\ 2 & 2 \\ 2 & 0 \\ 2 & 2 \\ 2 & 2 \\ 0 & 2 \\ 2 & 2\end{array}$

AIT
0
0
0
0
0
0
0
0
0
0
2
0
0
0
0
2
0
2
2

$\begin{array}{ll}E & \text { IVF } \\ 0 & 1 \\ 0 & 1 \\ 0 & 1 \\ 0 & 1 \\ 0 & 1 \\ 1 & 2 \\ 0 & 2 \\ 0 & 2 \\ 2 & 2 \\ 2 & 2 \\ 1 & 2 \\ & \\ 1 & 2 \\ 0 & 2 \\ 0 & 2 \\ 1 & 1 \\ 2 & 0 \\ 2 & 1 \\ 2 & 2 \\ 2 & 2 \\ & \end{array}$

A: Aleatorización: Sí 2, Parcial 1. GC: Grupo Control: Sí: 2, Pre-post 1.

AP: Análisis Preintervención: Sí 2, Parcial 1. S: Seguimiento-Pérdidas: No especificadas

o Elevadas 0, Especificadas y no elevadas 2. AIT: Análisis por Intención de Tratar: No 0; Sí:2

E: Enmascaramiento: Simple 1, Doble 2. IVF: Instrumento Válido y Fiable: ;Válido o Fiable 1;

Válido y Fiable 2. RE: Resultado Evaluado: Conducta del médico 1, satisfacción

Paciente 1, cualquier resultado de salud en paciente 2. Am: Ambito: Multicéntrico 2 Unicentrico 0.

dios el autor seleccionó y ponderó algunas características metodológicas consideradas importantes para la valoración de ensayos educativos (tabla 1).

\section{RESULTADOS}

Estudios realizados en poblaciones de Residentes

En la tabla 2 aparecen los 11 estudios experimentales o cuasi experimentales de intervenciones educativas en formación en entrevista clínica (EC) con residentes (RR), ordenados en función de su calidad metodológica. En la tabla 2 se especifican algunas de las características docentes valoradas. Tres de ellos muestran resultados negativos globa$\operatorname{les}^{13-15}$. Como características comunes más sobresalientes de estos tres destacan el que se realizaban en el tercer año de la residencia y se impartían a lo largo del tiempo: entre 30, 12 y 6 meses respectivamente. En el estudio de Thompson et al. ${ }^{13}$ y en el estudio COMCORD ${ }^{15}$ a diferencia del de Schubert et al. ${ }^{14}$ se utilizaron métodos de aprendizaje interactivo (es decir seguían una metodología de aprendiza- je basada en problemas y en la experiencia de los alumnos) y los dos primeros ${ }^{13,15}$ medían los resultados en la conducta (habilidades puestas en práctica) de los RR.

El resto de los ocho estudios comunicaban resultados positivos. De estos, excepto uno que se llevó a cabo a lo largo de 6 meses en 18 sesiones de $2 \mathrm{hs}^{16}$ todos eran cursos intensivos (1 mes a tiempo completo, generalmente, de más de $100 \mathrm{hs}$ ) en los que se producía una inmersión total del residente en el aprendizaje de la EC y dominios clínicos psicosociales. Los trabajos de Smith et al. ${ }^{17}$, Roter et al. ${ }^{18}$, Kauss ${ }^{19}$ y Gask et al. ${ }^{16}$ presentaban características similares, entre ellas la medición del cambio de conducta que se produce en los RR mediante pacientes reales $(\mathrm{PR}) \mathrm{o} / \mathrm{y}$ pacientes simulados (PS) o su efecto en pacientes. Estos cuatro estudios utilizan instrumentos de medida validados y fiables. La población de estos estudios son RR de 1er año (el de Kauss et al incluye también estudiantes). Todos tienen un marcado carácter experimental, siendo de buena 


\begin{tabular}{|c|c|c|c|c|c|c|c|c|c|}
\hline Autores/año & $\begin{array}{l}N^{\circ} \\
\mathrm{RR}\end{array}$ & $\begin{array}{l}\text { Tipo de } \\
\text { curso/hs }\end{array}$ & Año & $\begin{array}{l}\text { Actividad } \\
\text { Clínica }\end{array}$ & $\begin{array}{l}\text { Orientación Docente } \\
\text { Predominante }\end{array}$ & Monitores & $\begin{array}{l}\text { Medición } \\
\text { de conducta }\end{array}$ & $\begin{array}{l}\text { Medición } \\
\text { pacientes }\end{array}$ & RESULTADOS \\
\hline Thompson (13) & 21 & L/TP (30meses)/150 & $3^{\circ}$ & ¿? & $\mathrm{CA} / \mathrm{CP}$ & Ps & Sí & No & Negativo \\
\hline Schubert (14) & 24 & L/TP(6-12meses)/60-120 & $3^{\circ}$ & i? & $\mathrm{CP}$ & Ps & No/Registros & No & Negativo \\
\hline Kauss (19) & 69 & $\mathrm{I} / \mathrm{TC}(1 \mathrm{mes})$ & 1\%/Estudiantes & \begin{tabular}{|l|}
2 meses \\
en Atención \\
Primaria
\end{tabular} & $\begin{array}{l}\text { CP/CA: } \\
\text { Didácticas+práctica } \\
\text { + interacción PR+feedback } \\
\text { CP: Tarjetas informativas. }\end{array}$ & i? & Sí con PR & No & $\begin{array}{l}\text { Positivo } \\
\text { Empatía, respuestas } \\
\text { psicolsociales y } \\
\text { afectivas entre Int y Co } \\
\text { Negativo para } \\
\text { respuestas exploratorias } \\
\text { y entre los } 2 \text { de Int. }\end{array}$ \\
\hline Merkel (22) & 16 & I/TC/900 & $3^{\circ}$ & $\begin{array}{l}\text { Atención } \\
\text { Primaria }\end{array}$ & $\begin{array}{l}\text { CP/CA: } \\
\text { Seminarios, conferencias, } \\
\text { rotaciones en Psiquiatría } \\
\text { supervisadas, grupos Balint } \\
\text { y counselling }\end{array}$ & $\begin{array}{l}\text { Ps } \\
\text { NoM }\end{array}$ & $\begin{array}{l}\text { No/ } \\
\text { Registros y Q } \\
\text { autorellenos }\end{array}$ & Sí & $\begin{array}{l}\text { Positivo } \\
\text { Actitud, diagnósticos, } \\
\text { consejo y derivación } \\
\text { a Salud Mental. } \\
\text { Negativo Menos } \\
\text { satisfacción en pacientes }\end{array}$ \\
\hline Breulin (23) & 24 & I/TC/160 & $1^{\circ}$ & $\begin{array}{l}\text { mes } \\
\text { en Pediatría }\end{array}$ & $\begin{array}{l}\text { CA/CP: } \\
\text { Conferencias, seminarios, } \\
\text { Curso de } 26 \text { hs de ECi?? } \\
\text { Cintas video. Experiencias } \\
\text { Hospital } \\
\end{array}$ & $\begin{array}{l}\text { Ps } \\
\text { NoM } \\
\text { M }\end{array}$ & $\begin{array}{l}\text { No/Q } \\
\text { autorrellenos }\end{array}$ & No & $\begin{array}{l}\text { Positivo conocimientos } \\
\text { Negativo habilidades } \\
\text { ejecutivas y } \\
\text { observacionales }\end{array}$ \\
\hline Smith (20) & 48 & $\mathrm{I} / \mathrm{TC} / 120-160$ & $3^{\circ}$ & $\begin{array}{l}1 \text { mes } \\
\text { en Medicina } \\
\text { psicosocial }\end{array}$ & $\begin{array}{l}\text { CA/CP: } \\
\text { Seminarios, Entrevistas } \\
\text { a PR con feedback } \\
\text { de EC y Psicosocial. } \\
\text { Consultas con PR } \\
\text { psiquiatricos o estresados }\end{array}$ & $\begin{array}{l}\text { Ps } \\
\text { NoM } \\
\text { M }\end{array}$ & $\begin{array}{l}\text { No/Q } \\
\text { de actitudes } \\
\text { y habilidades }\end{array}$ & No & $\begin{array}{l}\text { Positivo conocimiento, } \\
\text { autovaloración, actitudes }\end{array}$ \\
\hline Smith (21) & 26 & T/TC/i? & $1^{\circ}$ & $\begin{array}{l}\text { mes } \\
\text { en medicina } \\
\text { psicosocial }\end{array}$ & $\begin{array}{l}\text { CA/CP: Visitas a Pa., } \\
\text { Seminarios: Conferencia, } \\
\text { demostración, role-model } \\
\text { \& play. Temas generales } \\
\text { y específicos }\end{array}$ & i? & $\begin{array}{l}\text { No/Q } \\
\text { sobre } \\
\text { conocimiento } \\
\text { y actitudes }\end{array}$ & No & $\begin{array}{l}\text { Positivo (En } 8 / 15 \\
\text { ítems) confianza en } \\
\text { sus habilidades } \\
\text { psicosociales, respuesta } \\
\text { emocional y manejo. } \\
\text { Somatización. } \\
\text { Conocimiento. } \\
\end{array}$ \\
\hline Roter (18) & 26 & I/TC/160hs & $1^{\circ}$ & $\begin{array}{l}1 \text { mes en } \\
\text { psiquiatria }\end{array}$ & $\begin{array}{l}\text { CA/CP: Didácticos, } \\
\text { Participación en consulta, } \\
\text { Role-plays PS y PR: } \\
\text { feedback }\end{array}$ & $\begin{array}{l}\text { Ps } \\
\text { NoM } \\
\text { M }\end{array}$ & $\begin{array}{l}\text { Sí con PS } \\
\text { (registros) }\end{array}$ & $\begin{array}{l}\text { Sí } \\
\text { (opinión } \\
\text { del PS) }\end{array}$ & $\begin{array}{l}\text { Positivo Preguntas } \\
\text { abiertas, menos } \\
\text { dirigidas, resúmenes } \\
\text { y counselling } \\
\text { Mejor valoración PS } \\
\text { Negativo: Contenido } \\
\text { preguntas. Registros. }\end{array}$ \\
\hline Smith (17) & 69 & $\mathrm{I} / \mathrm{TC} / \mathrm{i} ?$ & $1^{\circ}$ & $\begin{array}{l}1 \text { mes en } \\
\text { medicina } \\
\text { psicosocial }\end{array}$ & $\begin{array}{l}\text { CA/CP: Seminarios: } \\
\text { discusión demostración } \\
\text { y práctica role-play } \\
\text { Entrevistas con P: } \\
\text { feedback. Bibliografia } \\
\text { Temas específicos }\end{array}$ & $\begin{array}{l}\text { Ps } \\
\text { M }\end{array}$ & $\begin{array}{l}\text { Sí con PS } \\
\text { y PR (+Q) }\end{array}$ & $\begin{array}{l}\text { Sí } \\
\text { con } 238\end{array}$ & $\begin{array}{l}\text { Positivo Mejor } \\
\text { con PS: respuestas } \\
\text { a emoción, } \\
\text { información psicosocial } \\
\text { y centrados en Pa. } \\
\text { Negativo Satisfacción } \\
\text { y bienestar de los PR. } \\
\text { Tendencias + }\end{array}$ \\
\hline Gask (16) & 14 & $\begin{array}{l}\text { L/TP/6 } \\
\text { meses/36 hs }\end{array}$ & i? & $\begin{array}{l}\text { actividades } \\
\text { no definidas }\end{array}$ & $\begin{array}{l}\text { CA: Entrevistas } \\
\text { videógrabadas con } \\
\text { PR: feedback } \\
\text { Grupos pequeños }\end{array}$ & $\begin{array}{l}\mathrm{M} \\
\mathrm{Ps}\end{array}$ & 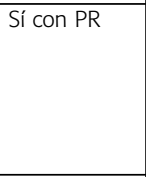 & No & $\begin{array}{l}\text { Positivo Detección } \\
\text { de problema } \\
\text { psiquiátrico. Definición } \\
\text { problema, consejos y } \\
\text { preguntas psicológicas } \\
\text { Empatía }\end{array}$ \\
\hline Ruiz Moral (15) & 193 & $\begin{array}{l}\text { L/TP/6- } \\
8 \text { meses/30 hs }\end{array}$ & $3^{\circ}$ & $\begin{array}{l}\text { Atención } \\
\text { primaria }\end{array}$ & $\begin{array}{l}\text { CA/CP: } \\
\text { Ejercicios experienciales } \\
\text { Práctica: role-play } \\
\text { Entrevistas PR: feedback } \\
\text { Prácticas en consulta } \\
\text { Bibliografía }\end{array}$ & $\mathrm{M}$ & Sí con PS & No & $\begin{array}{l}\text { Negativo Para } \\
\text { exploración del } \\
\text { contexto, búsqueda } \\
\text { de acuerdos } \\
\text { Positivo Para } \\
\text { organizarse la consulta }\end{array}$ \\
\hline
\end{tabular}

EC: Entrevista Clínica; RR: residentes; L:Iongitudinal,|lintensivo; TC:tiempo completo;TP:tiempo parcial;i?:no definido;CA:centrado en el alumno;CP:centrado en el profesor; Ps: psiquiatras-psicólogos;NoM: no médicos;M:médicos;Int:intervención;Co:control; Q:cuestionarios;PR:paciente real.

calidad, aunque no son multicéntricos y dos de ellos $^{17,18}$ no miden las habilidades comunicacionales previas de los RR. Los métodos de aprendizaje ensayados aquí son interactivos, especialmente el de Gask et al. que es muy similar al estudio español COMCORD. Los otros 4 trabajos con resultados positivos : los de Smith et al. '91 y ' $95{ }^{20,21}$, Merkel et al. ${ }^{22}$ y Breulin et al. ${ }^{23}$, no miden el impacto de los cursos en el cambio de conductas bien sea con PR y/o PS, sino conocimientos, predisposición, autoconfianza, otras actitudes, diagnósticos psicosociales, derivaciones a Salud Mental, etc. Esto los situaría en un nivel inferior a los anteriores. El de Merkel et al. ${ }^{22}$ (que no incluye $\mathrm{RR}$ aleatoriamente sino por su 
grado de motivación) aborda satisfacción de los pacientes y análisis de los registros médicos. Salvo uno de ellos ${ }^{20}$, en el que participan $28 \mathrm{RR}$ de $1 \mathrm{er}$ año, el resto se realizan con un escaso número: 26 , 24 y 16 respectivamente. Todos se caracterizan por ser periodos intensivos de aproximadamente un mes de rotatorio y enseñanza interactiva. En mayor o menor medida suelen emplear métodos docentes mixtos. De los más interactivos, son $\operatorname{dos}^{20,21}$ los que presentan mejores resultados. En el estudio de Breulin et al. $^{23}$ existen determinaciones preintervención y sólo es positivo para conocimientos. Como en el primer grupo de trabajos con resultados positivos, los instrumentos de medida utilizados en todos estos, que salvo excepciones suelen presentar indices psicométricos de validación incluyendo al valorador, suelen ser muy diferentes, siendo aptos para registrar distintas habilidades o actitudes.

\section{Estudios en educación médica continuada}

Ocho han sido los estudios de formación médica continuada recuperados, todos en el ámbito de la atención primaria. La tabla 1 los clasifica en función de su excelencia metodológica, en la tabla 3 se detallan los aspectos docentes más importantes evaluados. En estos casos los cursos y seminarios suelen ser intensivos realizados con una duración de entre 4 a 48 horas ${ }^{24,25,26,27,28,29,30}$, dos de ellos ${ }^{24,31}$ realizan sesiones longitudinales a lo largo de 3 y 5 meses por un total de 36 hs. Salvo los más antiguos ${ }^{24,25}$ la metodología es predominantemente centrada en el que aprende (interactiva y basada en la experiencia del aprendiz). Metodológicamente son de mayor calidad, casi todos incluyen mediciones previas a la intervención y los resultados miden cambios de conducta en los médicos con PR o PS y/o modificaciones en los pacientes. Los instrumentos de medida utilizados, que salvo excepciones suelen presentar indices psicométricos de validación, incluyendo al valorador, suelen ser también diferentes unos de otros. Todos resaltan en mayor o menor medida resultados positivos, detallados en la tabla 3 . Son varios los estudios que recogen modificaciones en el estado de salud de los pacientes en sentido positivo ${ }^{24,25,26,27,29,30}$.

\begin{tabular}{|c|c|c|c|c|c|c|c|c|c|}
\hline Autores/año & $\begin{array}{l}N^{\circ} \\
M\end{array}$ & $\begin{array}{l}\text { Tipo de } \\
\text { curso/hs }\end{array}$ & Evaluación & $\begin{array}{l}\text { Mediciones/ } \\
\text { Tiempo }\end{array}$ & $\begin{array}{l}\text { Orientación } \\
\text { y Técnicas }\end{array}$ & Monitores & $\begin{array}{l}\text { Medición } \\
\text { de conducta }\end{array}$ & $\begin{array}{l}\text { Medición } \\
\text { pacientes }\end{array}$ & RESULTADO \\
\hline Inui (24) & 62 & $\begin{array}{l}\text { 1/2 sesiones: } 4 \text { hs } \\
\text { en } 3 \text { meses }\end{array}$ & Q & $\begin{array}{l}\text { Registros en } \\
\text { M. En Pa: } \\
\text { Ideas sobre } \\
\text { HTA, eficacia } \\
\text { y adherencia } \\
\text { a Tto y } \\
\text { control } 1 \text { mes } \\
\end{array}$ & $\begin{array}{l}\text { CP: Taller sobre } \\
\text { conocimientos y } \\
\text { problemas de adherencia } \\
\text { Modelo de creencias. } \\
\text { Entrevista basada en } \\
\text { actitudes y percepciones }\end{array}$ & ¿? & $\begin{array}{l}\text { No/Q y } \\
\text { Registros }\end{array}$ & $\begin{array}{l}\text { Sí } \\
\text { (PR:109) }\end{array}$ & $\begin{array}{l}\text { Positivo Conocimiento, } \\
\text { adherencia y } \\
\text { control. Tensión }\end{array}$ \\
\hline Evans (25) & 40 & I/2 sesiones: 3 hs: $6 \mathrm{hs}$ & $\begin{array}{l}\mathrm{Q} / \mathrm{P} \\
\text { ciego }\end{array}$ & \begin{tabular}{l|} 
Ansiedad, \\
tipo información \\
claridad, \\
extensión. \\
Simpatía \\
Satisfacción \\
\end{tabular} & \begin{tabular}{|l|} 
CP \\
Conferencia y \\
discusión entre M solo \\
\end{tabular} & i? & $\begin{array}{l}\mathrm{No} / \mathrm{Q} \\
\end{array}$ & $\begin{array}{l}\text { Sí } \\
\text { (PR:400) }\end{array}$ & $\begin{array}{l}\text { Positivo Satisfacción } \\
\text { menos ansiedad } \\
\text { tras consulta. } \\
\text { Escasas diferencias } \\
\text { pero significativas }\end{array}$ \\
\hline Bowman (31) & 10 & L/5 meses: $36 \mathrm{hs}$ & Q/ ciego & & $\begin{array}{l}\text { CA Entrevistas } \\
\text { videógrabadas con } \\
\text { PR: feedback } \\
\text { Grupos pequeños }\end{array}$ & Ps & Sí con Ps & No & $\begin{array}{l}\text { Positivo Respuesta } \\
\text { a claves verbales. } \\
\text { A largo plazo (hasta } \\
22 \text { meses): menos } \\
\text { preguntas de checklist, } \\
\text { más checking-out. }\end{array}$ \\
\hline Levison (26) & 53 & $\begin{array}{l}\text { Corto: } 4,5 \text { hs } \\
\text { Largo: } 2,5 \text { dias }\end{array}$ & $>1 / Q / i ?$ & $\begin{array}{l}\text { Preguntas } \\
\text { Información } \\
\text { que los Pa daban } \\
\text { distres de los P, } \\
\text { Tiempo de habla } \\
\text { y tipo (PS, BM), } \\
\text { expresiones de } \\
\text { afecto }\end{array}$ & $\begin{array}{l}\text { Corto: CP: didática, dicusión } \\
\text { casos. Largo: CA: interés } \\
\text { personal, repetición, } \\
\text { vídeo + feedback } \\
\end{array}$ & ¿? & Sí con PR & $\begin{array}{l}\text { Sí en } \\
473 \text { PR }\end{array}$ & $\begin{array}{l}\text { Negativo el taller corto } \\
\text { Positivo el curso largo } \\
\text { M: preguntas abiertas, } \\
\text { Preguntar la opinión de } \\
\text { los Pa, información BM } \\
\text { Pa: exponían más } \\
\text { información BM y } \\
\text { psicosocial, menos } \\
\text { expresiones negativas } \\
\text { en M y Pa, menos } \\
\text { signos de distress. }\end{array}$ \\
\hline Roter (27) & 69 & 1/8 hs/2 semanas & $\begin{array}{l}>1 / Q / \\
\text { no ciego }\end{array}$ & $\begin{array}{l}\text { Manejo CA } \\
\text { emoción/ } \\
\text { detección } \\
\text { problema/salud } \\
\text { en } \mathrm{P}\end{array}$ & $\begin{array}{l}\text { CP/CA } \\
\text { Didácticos, PS, PR, } \\
\text { feedback/Inmediata y en } \\
\text { PR hasta } 6 \text { meses }\end{array}$ & i? & $\begin{array}{l}\text { Sí con PR y } \\
\text { PS }\end{array}$ & $\begin{array}{l}\text { Sí en } \\
648\end{array}$ & $\begin{array}{l}\text { Positivo Mejor ME } \\
\text { y DP en cada grupo } \\
\text { con PR y PS (Sacar } \\
\text { información, } \\
\text { expectativas, AVE, } \\
\text { preocupaciones) } \\
\text { Mejorar distress en PR } \\
\text { todos pero más el DP) }\end{array}$ \\
\hline
\end{tabular}




\begin{tabular}{|c|c|c|c|c|c|c|c|c|c|}
\hline Autores/año & $\begin{array}{l}\mathrm{N}^{\circ} \\
\mathrm{M}\end{array}$ & $\begin{array}{l}\text { Tipo de } \\
\text { curso/hs }\end{array}$ & Evaluación & $\begin{array}{l}\text { Mediciones/ } \\
\text { Tiempo }\end{array}$ & $\begin{array}{l}\text { Orientación } \\
\text { y Técnicas }\end{array}$ & Monitores & $\begin{array}{l}\text { Medición } \\
\text { de conducta }\end{array}$ & $\begin{array}{l}\text { Medición } \\
\text { pacientes }\end{array}$ & RESULTADO \\
\hline Girón (28) & 20 & L/48 hs en 7 semanas & 1/Q/ciego & $\begin{array}{l}\text { Contacto visual } \\
\text { Postura } \\
\text { facilitaciones no } \\
\text { verbal/verbal } \\
\text { interrupciones, } \\
\text { preguntas } \\
\text { psicológo al mes }\end{array}$ & $\begin{array}{l}\text { CP/CA } \\
\text { Didácticas, } \\
\text { Repetición role-play } \\
\text { y feedback }\end{array}$ & ¿? & Sí con PR & No & $\begin{array}{l}\text { Positivo Contacto, } \\
\text { visual, Postura, } \\
\text { facilitaciones no } \\
\text { verbales } \\
\text { Negativo Facilitaciones } \\
\text { verbales, interrupciones } \\
\text { preguntas psicológicas }\end{array}$ \\
\hline Sanci (32) & 108 & L/15 hs en 6 semanas & 1/Q/ciego & $\begin{array}{l}\text { Habilidades } \\
\text { Competencia } \\
\text { autopercibida } \\
\text { Conocimiento } \\
1 \text { año }\end{array}$ & $\begin{array}{l}\mathrm{CA} / \mathrm{CP} \\
\text { Talleres de 2,5 hs: } \\
\text { Didácticas, actividades } \\
\text { basadas en problemas } \\
\text { Role model\&play } \\
\text { PS+feedback }\end{array}$ & $\begin{array}{l}\text { M, NoM\& } \\
\text { Ps }\end{array}$ & Sí con PS & No & $\begin{array}{l}\text { Positivo Habilidades: } \\
\text { Satisfacción, Relación, } \\
\text { Confidencialidad, } \\
\text { Competencia, valoración } \\
\text { riesgo, Competencia, } \\
\text { autopercibida y } \\
\text { conocimiento } \\
\end{array}$ \\
\hline $\begin{array}{l}\text { Muñoz } \\
\text { Alamo(28) } \\
\text { y Ruiz Moral (29) }\end{array}$ & 20 & l/18 hs en 1 semanas & 1/Q/ciego & $\begin{array}{l}\text { Grado CP/ } \\
\text { inmediata y en } \\
\text { un año }\end{array}$ & $\begin{array}{l}\text { CP/CA } \\
\text { Didácticas, } \\
\text { Role model\&play } \\
\text { PS + feedback }\end{array}$ & $M$ & $\begin{array}{l}\text { Sí con PS } \\
\text { y PR }\end{array}$ & $\begin{array}{l}\text { Sí en } \\
110 \text { PR }\end{array}$ & $\begin{array}{l}\text { Positivo Explorar, contexto } \\
\text { discusión amplia, } \\
\text { acuerdos. En PR: dolor, } \\
\text { ansiedad, puntos } \\
\text { dolorosos, movilidad }\end{array}$ \\
\hline
\end{tabular}

L: longitudinal; I: intensivo; Ps: psiquiatras-psicólogos; NoM: no médicos, M: médicos; Q: cuestionarios; EC: entrevista clínica; PR: paciente real; PS: paciente simulado; Pa: pacientes; ¿?: no definido; CA: centrado en el alumnado; CP: centrado en el profesor; ME: manejo de emoción; DP: detección del problema; BM: biomédico; AVE: acontecimientos vitales estresantes.

La medición de los resultados sin embargo, suele ser en casi todos inmediata a la intervención (salvo en los estudios de Bowman et al. ${ }^{31}$, Roter et al. ${ }^{27}$ Ruiz y Muñoz ${ }^{29,30}$ y Sanci et al.$^{32}$ que la medición se prolonga 2,6 y hasta 1 año en los dos últimos). Por regla general los médicos que participan en estos estudios suelen ser médicos motivados en mejorar su estilo de consulta o introducir nuevas habilidades, esto es especialmente claro en los estudios de Bowman et al. ${ }^{31}$ (que ya habían recibido un curso similar en su periodo de residencia), Muñoz y Ruiz. ${ }^{29,30}$ y Sanci et al. ${ }^{32}$ (que respondieron a la oferta de realizar gratis un curso sobre atención a adolescentes). Esto los diferencia de los estudios que se realizan con $R R$ como parte de su programa de formación.

\section{Estudios con pregraduados}

Respecto a los estudios realizados en estudiantes de medicina (tabla 4), hemos analizado 5 de características experimentales. En general no se puede decir que estos estudios comparen programas formativos sino determinadas técnicas docentes, particularmente técnicas centradas en el que aprende, de las que el feedback sobre interacciones de los estudiantes con pacientes (simulados o reales) es la más estudiada. Suelen realizarse en el ámbito de rotación práctica de entre 2 a 8 semanas de los estudiantes en especialidades de atención primaria (incluida salud mental y pediatría). Todos miden el resultado en la conducta de los estudiantes al enfrentarse a un PS o PR y salvo un estudio ${ }^{33}$ todos los demás comunican resultados positivos ${ }^{34-37}$, si bien uno de ellos ${ }^{35}$ no encuentra diferencias entre feedback y autoinstrucción con un vídeo. Por regla general los resultados positivos son con habilidades muy básicas del tipo de atender al paciente: mirarlo inclinarse, repetición de sus sentimientos o mensajes ${ }^{37}$, respuesta apropiada a problemas adicionales, a temas sensibles, cierre ${ }^{36}$ y recogida de historia ${ }^{34,36}$, modales profesionales, relación interprofesional ${ }^{35} \mathrm{y}$ dar información ${ }^{34,35}$. Una vez más los instrumentos de medición utilizados suelen ser variados.

\section{DISCUSIÓN}

En esta selección predominan los estudios con resultados positivos; seguramente existe un sesgo de publicación hacia estos en comparación con los negativos, pero sin un análisis de la "literatura gris" no es posible comprobarlo. Aunque los estudios seleccionados por regla general han utilizado instrumentos validados y fiables, el mayor inconveniente que tienen el análisis y comparación de los resultados de los distintos trabajos es que cada uno de ellos emplea instrumentos de evaluación diferentes lo que, generalmente, implica el reflejar distintas facetas de la interacción médica. Este problema ha llevado a algunos autores a sugerir que los investigadores y clínicos deberían coordinar los esfuerzos e intercambiar los diferentes materiales videograbados de los que dispone cada uno para ser reanalizados con los distintos instrumentos ${ }^{38}$.

Metodológicamente llama la atención la escasez de estudios multicéntricos, lo que hace más controlables los factores confundentes, sin embargo, esto les dota de una menor capacidad para generalizar sus resultados. Por regla general aspectos como: 1. buenos diseños, 2. mediciones de la conducta, 3. cursos o seminarios intensivos, 4. realizarse estos en el contexto de una rotación o práctica clínicas relacionada con los contenidos y habilidades ensayados en el 
curso, y 5. metodologías interactivas entre las que se encuentra el ofrecer feedback sobre interacciones videograbadas con PR o PS (metodologías basadas en los principios de la enseñanza de adultos), suelen tener repercusión positiva en una amplia y variada gama de habilidades. Estas habilidades irían desde las más básicas en estudiantes (como por ejemplo, mirar a los ojos, mostrar modales correctos) hasta otras más complejas en alumnos con más experiencia clínica (como, mostrar empatía, o contención emocional en momentos difíciles). La metodología interactiva ha demostrado su eficacia frente a la tradicional o didáctica para el cambio efectivo de la conducta del médico o para influir positivamente en resultados de la consulta en otros ámbitos distintos al de la entrevista clínica ${ }^{39}$. El predominio de resultados positivos parece relacionarse también con un comienzo temprano en la formación (tanto en estudiantes como en RR de primeros años); el éxito de los cursos en médicos en ejercicio podría estar en gran medida condicionado por la motivación de éstos. Respecto a la composición del equipo docente, aunque equipos multidisciplinares han demostrado buenos resultados, un aspecto que estos estudios no valoran es en qué medida el facilitador o profesor influye, seduce o motiva al alumno, algo que quizás tenga una influencia decisiva.

Atendiendo a los resultados de esta revisión, en base a las recomendaciones realizadas por algunos de los consensos antes mencionados y en función de nuestra experiencia docente e investigadora en esta área ${ }^{15,29,30}$, exponemos a continuación algunos de los principios que de manera genérica sería interesante tener en cuenta actualmente en nuestro ámbito (for- mación postgraduada de especialidades clínicas) y en nuestro pais para conseguir una mayor eficacia en la formación en materia de comunicación clínica y para facilitar el desarrollo de las recomendaciones propuestas por los distintos grupos en cuanto a contenidos competenciales en comunicación clínica:

- Modificar adecuadamente los curricula, y las exigencias académicas para introducir desde el pregrado la formación en comunicación clínica

- Establecer de forma temprana en el periodo de residencia programas docentes para todos los residentes de carácter básico.

- Una vez hecho lo anterior, la introducción de habilidades en comunicación clínica más específicas y complejas (negociación, temas especiales como: malas noticias, somatización, paciente alcohólico, entrevista con familias, con adolescentes, etc) debe hacerse de forma escalonada durante la residencia para los residentes de medicina de familia y en casos concretos en función de cada especialidad.

- Los programas deben de ser muy específicos y bien estructurados con objetivos en los ámbitos cognitivos, de habilidades y actitudes bien delimitados, que, sin embargo, permitan atender las necesidades particulares de cada alumno.

- Independientemente de las recomendaciones generales competenciales de los programas docentes, estos deberían estar muy adaptados a las condiciones locales de cada ámbito docente teniendo en cuenta todos los recursos disponibles, especialmente el profesorado y tratando de elaborar sus propios objetivos en función de estos recursos.

- El personal docente debe estar, sobre todo sufi-

\begin{tabular}{|c|c|c|c|c|c|c|c|c|c|c|c|}
\hline Autores/año & $\begin{array}{l}N^{\circ} \\
\text { Est }\end{array}$ & $\begin{array}{l}\text { Tipo de } \\
\text { rotatorio/hs }\end{array}$ & $\begin{array}{l}\text { Año } \\
\text { E }\end{array}$ & $\begin{array}{l}\text { Modalidad } \\
\text { Docente }\end{array}$ & $\begin{array}{l}\text { Orientación } \\
\text { Predominante }\end{array}$ & Monitores & Diseño & $\begin{array}{l}\text { Situación pre/ } \\
\text { diferencias }\end{array}$ & $\begin{array}{l}\text { Medición de } \\
\text { conducta }\end{array}$ & $\begin{array}{l}\text { Medición } \\
\text { en pacientes }\end{array}$ & RESULTADOS \\
\hline Maguire (34) & 78 & $\begin{array}{l}4 \text { semanas } \\
\text { psiquiatría/i? }\end{array}$ & ¿? & $\begin{array}{l}\text { Didáctica }+ \\
\text { feedback por } \\
T V \text {, audio y } \\
\text { rating }\end{array}$ & $\mathrm{CA} / \mathrm{CP}$ & $M$ & $\begin{array}{l}\text { E/con } 4 \\
\text { grupos }\end{array}$ & $\begin{array}{l}\text { Si/No } \\
\text { difieren }\end{array}$ & Sí (con PR) & No & $\begin{array}{l}\text { Positivo } \\
\text { Técnicas, } \\
\text { información } \\
\text { cumplimentación }\end{array}$ \\
\hline Brown (33) & 62 & $\begin{array}{l}6 \text { semanas } \\
\text { pediatría/i? }\end{array}$ & 3 & $\begin{array}{l}\text { Didáctica } \\
3 \text { hs } \\
+ \text { feedback }\end{array}$ & CA/CP & varios & $\begin{array}{l}\text { E/con 3 } \\
\text { grupos }\end{array}$ & $\begin{array}{l}\text { Si/ difieren } \\
\text { a favor de Int. }\end{array}$ & Sí (con PS) & No & $\begin{array}{l}\text { Negativo } \\
\text { (el tiempo } \\
\text { es lo que } \\
\text { influye) }\end{array}$ \\
\hline Wheis (37) & 32 & $\begin{array}{l}\text { Medicina } \\
\text { General/10 } \\
\text { semanas/30 hs }\end{array}$ & s & $\begin{array}{l}\text { Didáctico } \\
\text { Demostración } \\
\text { Role-play } \\
\text { feedback }\end{array}$ & $\mathrm{CA} / \mathrm{CP}$ & $\mathrm{M}$ & $\begin{array}{l}\text { E/con 2 } \\
\text { grupos }\end{array}$ & Si/ No difieren & Sí (con PS) & No & $\begin{array}{l}\text { Positivo } \\
\text { en habilidades } \\
\text { básicas }\end{array}$ \\
\hline Scheidt (36) & 105 & $\begin{array}{l}\text { Pediatría/ } \\
2 \text { semanas/ } \\
\text { ¿? }\end{array}$ & 3 & $\begin{array}{l}\text { Feedback con } \\
\text { check points+ } \\
\text { didáctica }\end{array}$ & $\mathrm{CA} / \mathrm{CP}$ & $\begin{array}{l}\text { M } \\
\text { Pediatria }\end{array}$ & $\begin{array}{l}\text { QE/con 3 } \\
\text { grupos }\end{array}$ & No & Sí (con PR) & No & $\begin{array}{l}\text { Positivo } \\
\text { para el feedback } \\
\text { en ciertas técnicas } \\
\text { de EC y exploración }\end{array}$ \\
\hline Mason (35) & 60 & $\begin{array}{l}2 \text { meses en } \\
\text { Medicina } \\
\text { General/i? }\end{array}$ & 4 & $\begin{array}{l}\text { Material } \\
\text { audiovisual, } \\
\text { autoinstructivo } \\
\text { y feedback }\end{array}$ & CA/CP & $\begin{array}{l}\text { Ps } \\
\text { Pediatria } \\
\text { M }\end{array}$ & $\begin{array}{l}\text { E/con } 4 \\
\text { grupos }\end{array}$ & Sí/ No difieren & Sí (con PS) & No & $\begin{array}{l}\text { Positivo } \\
\text { para la } \\
\text { autoinstrucción: } \\
\text { modales, relación } \\
\text { dar información }\end{array}$ \\
\hline
\end{tabular}

Ps: psiquiatras-psicólogos; NoM: no médicos; M: médicos; Int: intervención; Co: control; Q: cuestionarios; EC: entrevista clínica; E: experiemental; QE: cuasi-experimental; PR: paciente real, PS: paciente simulado; Pa: pacientes; i?: no definido; CA: centrado en el alumno; CP: centrado en el profesor. 
cientemente capacitado y a veces es preferible que sea multidisciplinar (esto suele depender del tema que se trate). Por lo que, dada la escasez del mismo, un objetivo prioritario debería ser la capacitación de docentes y su acreditación fiable.

- Parece más adecuado incluir estos programas de forma más o menos intensiva en periodos no demasiado prolongados y en los que el contexto, es decir, las actividades clínico-asistenciales estén estrechamente relacionadas con estos seminarios y cursos o con otras actividades complementarias en el área de comunicación clínica y medicina psicosocial. Es decir deberían estar en sintonía con la realidad clínica cotidiana.

- El simultanear actividades de este tipo de cursos y seminarios con otras diferentes, exigen a los $\mathrm{RR}$ una atención dividida que parece contribuir a diluir su efectividad.

- La metodología docente para intentar el cambio de comportamientos ha de estar fundamentada en los métodos de aprendizaje de adultos: basado en los problemas que les son significativos y en su experiencia clínica práctica, es decir una formación centrada en el residente.

- Es preciso promover la investigación y la evaluación de estas y otras estrategias docentes con perspectivas tanto cualitativas como cuantitativas

Finalmente, la comunicación médico-paciente ha sido conceptualizada bien como una "habilidad" más o como un "estado de relación" ${ }^{40}$. Ambas aproximaciones son diferentes desde el punto de vista del análisis y la investigación. La aproximación a la competencia comunicativa enfocada en las habilidades se apoya en las conductas observadas. La aproximación que presenta la comunicación como una forma de "relacionarse" más globalizadora intenta valorar los efectos de la misma tal y como son experimentados por sus propios protagonistas. Esta distinción es importante tanto para la docencia como para la investigación. La presente revisión de los trabajos sigue el primer enfoque por lo que sus conclusiones son necesariamente incompletas ya que la enseñanza puede y debe enfocarse tanto a la modificación de conductas como al desarrollo personal del aprendiz, esto último implica que este tipo de enseñanza debe estar ligada a una formación ética y humana del médico que incluya el autoconocimiento y la promoción de valores genuinos en la atención a pacientes y familias.

\section{AGRADECIMIENTOS}

Quiero expresar $\mathrm{mi}$ agradecimiento al $\mathrm{Dr}$

Francesc Borrell Carrió, Coordinador Estatal del
Grupo Comunicación y Salud y al Dr Juan José Rodríguez Salvador, Coordinador del Grupo Comunicación y Salud del Pais Vasco y representante español en el Comité del EACH por las valiosas sugerencias que han enriquecido el manuscrito original.

\section{REFERENCIAS}

1. Laín Entralgo P. La relación médico-enfermo. Madrid: Alianza; 1983.

2. Engel G. The need for a new medical model: a challenge for biomedicine. Science 1977;196:129-36.

3. Stewart M, Brown J, H B, Galajda J, Meredith L, Sangster M. Evidence on patient-doctor communication. Can Preven Control 1999;3:25-30.

4. Simpson M, Buckman R, Stewart M, Maguire P, Lipkin $\mathrm{M}$, Novack D, et al. Comunicación médico-paciente: el informe del consenso de Toronto. BMJ (ed esp) 1993;8:40-5.

5. General Medical Council. Tomorrow's doctors. Recommendations on undergraduate medical education. London: General Medical Council; 1993.

6. Association of American Medical Colleges. Contemporary issues in medicine: Communication in medicine. Washington, DC: AAMC; 1999 October. Report No.: III.

7. Consensus statement from the workshop on the teaching and assessment of communcation skills in Canadian medical schools. Can Med Assoc J 1992;147:1149-52.

8. Makoul G, Schoefield T. Communication teaching and assessment in medical education: an international consensus statement. Patient Education and Counseling 1999;137:191-5.

9. Participants in the Bayer-Fetzer Conference on PhysicianPatient Communication in Medical Education. Essential Elements of Communication in Medical Encounters: The Kalamazoo Consensus Statement. Acad Med 2001;76: 390-393.

10. Comisión Nacional de la Especialidad de Medicina Familiar y Comunitaria. Programa Nacional de la Especialidad de Medicina de Familia y Comunitaria. Madrid: Ministerios de Sanidad y Consumo y de Educación y Ciencia; 1993.

11. Grupo semFYC Comunicación y Salud. Competencias del Médico de Familia. IA. La comunicación asistencial. La entrevista Clínica. La relación médico-paciente. Madrid, 2002.

12. Grupo de Trabajo de la Consejería de Salud de la Junta de Andalucía. Formación común para los especialistas en formación del sistema sanitario público de Andalucía. Módulo II: Entrevista clínica y relación médico-paciente. Sevilla: Consejería de Salud de la Junta de Andalucía; 2002.

13. Thompson T, Stoudemire A, Mitchell W. Effects of a 
psychiatric liaison program on internists' ability to assess psychosocial problems. Int J Psychiatry Med 1982;12:153-60.

14. Schubert D, Billowitz A, Gabinet L, Friedson W. Effect of liaison psychiatry on attitudes toward psychiatry, rate of consultation, and psychosocial documentation. Gen Hosp Psychiatry 1989;11:77-78.

15. Ruiz Moral R, Rodríguez Salvador J, Pérula de Torres L, Prados Castillejo JA. Effectiveness of a training program in interviewing for family medicine trainees: the COMCORD project. Fam Med 2003; 35:489-95.

16. Gask L, Goldberg D, Lesser A, Millar T. Impro-ving the psychiatric skills of the general practice trainee: an evaluation of a group training course. Med Educ 1988;22:132-8.

17. Smith R, Lyles J, Mettler J, Stoffelmayr B, Van Egeren L, Marshall A, et al. The effectiveness of intensive training for residents in interviewing. A randomized, controlled study. Ann Intern Med 1998;128:118-126.

18. Roter D, Cole K, Kern D, Barker L, Grayson M. An evaluation of residency training in interviewing skills and the psychosocial domain of medical practice. J Gen Intern Med 1990;5:347-54.

19. Kauss D, Robbins A, Heinrich R, Abrass I. Interpersonal skills training: comprehensive approach versus brief instruction. J Med Educ 1981;56:663-5.

20. Smith R, Osborn G, Hoppe R, Lyles J, Van Egeren L, Henry R, et al. Efficacy of a one-month training block in psychosocial medicine for residents: a controlled study. J Gen Intern Med 1991;6:535-43.

21. Smith R, Mettler J, Stöffelmayr B, Lyles J, Marshall A, Van Egeren L, et al. Improving residents' confidence in using psychosocial skills. J Gen Intern Med 1995;10:315-20.

22. Merkel W, Nierenberg B. Behavioral science training in family practice residency education: a first evaluation. Soc Sci Med 1983;17:213-7.

23. Breunlin D, Richman J, Lattimer A. An evaluation strategy for behavioral pediatrics training. Fam Syst Med 1990;8:48-56.

24. Inui T, Yourtee E, Williamson J. Improved outcomes in hypertension after physician tutorials. A controlled trial. Ann Intern Med 1976;84:646-51.

25. Evans B, Kiellerup F, Stanley R, Burrows G, Sweet B. A communication skills programms for increasing patients' satisfaction with general practice consultations. Br J Med Psychol 1987;60:373-8.

26. Levinson W, Roter D. The effects of two continuing medical education programs on communication skills of practicing primary care physicians. J Gen Intern Med 1993;8:318-24.
27. Roter D, Hall J, Kern D, Barker R, Cole K, Roca R. Improving physicians' interviewing skills nd reducing patients' emotional distress. A randomized clinical trial. Arch Intern Med 1995;155:1877-84.

28. Girón M, Sánchez García E. Eficacia de una técnica de adiestramiento sobre las destrezas de entrevista clínica asociadas con la capacidad psicodiagnóstica del médico. Aten Primaria 1998; 21:125-30.

29. Ruiz Moral R, Muñoz Alamo M, Alba Jurado M, Pérula de Torres L. Effectiveness of a learner-centered training programme for primary care physicians in using a patientcentred consultation style. Fam Pract 2001;18:60-3.

30. Muñoz Alamo M, Ruiz Moral R, Pérula de Torres L. Evaluation of a patient-centred approach in generalized musculoskeletal chronic pain/ fibro-myalgia patients in primary care. Pat Edu Couns 2002;48:23-31.

31. Bowman F, Goldberg D, Millar T, Gask L, McGrath G. Improving the skills of stablished general practitioners: the long-term benefits of group teaching. Med Educ 1992;26:63-8.

32. Sanci L, Coffey C, Veit F, Carr-Gregg M, Patton G, Day N, et al. Evaluation oof the effectiveness of an educational intervention for general practitioners in adolescent health care: randomised controlled trial. BMJ 2000;320:224-30.

33. Brown J, O'Shea J. Improving medical student interviewing skills. Pediatrics 1980;65:575-8.

34. Maguire P, Roe P, Goldberg D, Jones S, Hyde C, O’Dowd $\mathrm{T}$. The value of feedback in teaching interviewing skills to medical students. Psychol Med 1978;8:695-704.

35. Mason J, Barkley S, Kappelman M, Carter D, Beachy W. Evaluation of a self-instructional method for improving doctor-patient communication. J Med Educ 1988;63:629-35.

36. Scheidt P, Lazoritz S, Ebbeling W, Figelman A, Moessner H, Singer J. Evaluation of system providing feedback to students on videotaped patient encounters. J Med Educ 1986;61:585-90.

37. Weihs K, Chapados J. Interviewing skills training -a study. Soc Sci Med 1986;23:31-4.

38. Boon H, Stewart M. Patient-physician communication assessment instruments: 1986 to 1996 in review. Pat Educ Couns 1998;35:161-76.

39. Davis D, Thomson O'Brien M, Freemantale N, Wolf F, Mazmanian P, Taylor-Vaisey A. Impact of formal continuing medical education. Do conferences, workshops, rounds, and other traditional continuing education activities change physician behabior or health care outcomes? JAMA 1999; 282:867-74.

40. Zoppi K, Epstein R. Is communication a skill? Communication behaviors and being in relation. Fam Med 2002;34:319-24. 